\title{
Um Mapeamento Sistemático para Problem Based Learning aplicado à Ciência da Computação
}

\author{
Armanda M. C. de A. Oliveira ${ }^{1}$, Rodrigo Lins Rodrigues ${ }^{2}$, Vinicius C. Garcia ${ }^{1}$ \\ ${ }^{1}$ Centro de Informática - Universidade Federal de Pernambuco \\ ${ }^{2}$ Departamento de educação - Universidade Federal Rural de Pernambuco \\ amcao@cin.ufpe.br, rlr@ded.ufrpe.br, vcg@cin.ufpe.br
}

\begin{abstract}
Studies on Problem-Based Learning, specifically in the area of Computer Science, need more evidence, especially in the use of learning disciplines of software engineering. Given this context, this work aimed to study a systematic mapping in order to identify studies involving the practice of PBL in the teaching of Computer Science. For this was used a methodology of searches performed on search engine in the relevant area of Informatics and Computer Science. From the analysis, the study concluded that most PBL applications were focused on the teaching of subjects such as Programming, Software Engineering and Operating Systems, and the use of hybrid methodologies with the use of Games in PBL and other methodologies to support PBL, as well as the development of applications and use of tools and technologies.
\end{abstract}

Resumo. Estudos que envolvem Aprendizagem Baseada em Problemas, especificamente na área de Ciência da Computação, necessitam de mais evidências, principalmente no que tange a utilização da aprendizagem de disciplinas da área de engenharia de software. Diante deste contexto, este trabalho teve como objetivo um estudo de mapeamento sistemático com a finalidade de identificar os estudos que envolvem a prática de PBL com o ensino da Ciência da Computação. Para tal foi utilizado uma metodologia de buscas realizadas em engenhos automatizados, relevantes na área de Informática e Ciência da Computação. A partir da análise o estudo concluiu que grande parte das aplicações de PBL foram voltadas para o ensino de disciplinas como Programação, Engenharia de Software e Sistemas Operacionais, e a utilização de metodologias híbridas com PBL fazendo o uso de Games, e outras metodologias de apoio a PBL, como também construção de aplicativos e uso de ferramentas e tecnologias.

\section{Introdução}

Para ter sucesso na área da Ciência da Computação, é necessário ter conhecimento e dominar uma variedade de habilidades, tais como a facilidade com matemática, lógica, resolução de problemas, pensamento algorítmico e programação. Infelizmente muitos estudantes têm dificuldades para desenvolver essas habilidades, principalmente quando o assunto é relacionado à programação orientada a objetos, matemática discreta, estrutura de dados e análise de algoritmos (MARTIN e CHINN, 2005). Tais problemas 
ocorrem pela maneira na qual o ensino é abordado nos cursos de graduação, visto que é possível notar uma grande falta de prática após a teoria (SANTOS, BATISTA, et al., 2010). A prática após a teoria permite com que os alunos façam conexões, ligando o conhecimento prévio aos novos conhecimentos aprendidos durante o novo processo de aprendizagem (SCHMIDT, 1993) e (COLES, 1991).

A partir destes problemas, e de uma busca para a melhor capacitação profissional, está sendo utilizado na área da Ciência da Computação um método educacional para o ensino da Computação, Problem Based Learning (PBL), ou Aprendizagem Baseada em Problemas. Essa metodologia ganhou aceitação e está se tornando cada vez mais eficaz dentro de uma variedade de disciplinas no ensino superior (YATES e GERDES, 1996).

A aprendizagem baseada em problemas faz uso dos problemas identificados para iniciar, motivar e focar a aquisição de conhecimentos, além de estimular o desenvolvimento de habilidades e atitudes no âmbito profissional. É considerada como sendo uma estratégia educacional centrada ao aluno, que o auxilia no desenvolvimento do raciocínio e comunicação, habilidades essenciais para o sucesso em sua vida profissional (ANDRADE, et al., 2007).

Essa metodologia surgiu na década de 70, através do médico Howard Barrows, sendo aplicada em turmas de medicina (DESLISLE, 1997). Era um método totalmente voltado para a área de saúde e hoje em dia vem tendo aceitação no ensino de diversas áreas do conhecimento, principalmente na Computação, e em outros níveis educacionais, fundamental e médio. No entanto os registros bibliográficos relacionados à PBL e Computação são poucos, e segundo (SAVIN-BADEN, 2000) esses estudos precisam de relatos dos atores principais do processo de ensinoaprendizagem, os alunos e professores. As pesquisas existentes tem se concentrado na aprendizagem e no desempenho dos alunos, poucas exploram as possibilidades de PBL, principalmente na área da Computação.

Para fornecer uma ampla visão desses estudos, e também pesquisar por estudos que tragam relatos sobre possibilidades de PBL, desafios e outros assuntos afins, é apropriado realizar um Mapeamento Sistemático. O Mapeamento Sistemático que tem como objetivo identificar a quantidade, tipo de pesquisa e resultados disponíveis dentro da área, além de verificar a evolução dos estudos na área de pesquisa escolhida (PETERSEN, FELDT, et al., 2007). Diante deste contexto, este trabalho tem como objetivo a realização de um Mapeamento Sistemático identificando indícios que venham a tornar a prática e aplicação da PBL mais conhecida e efetiva na área da Computação.

\section{Revisão bibliográfica}

Nesta seção é apresentada a revisão bibliográfica necessária para o desenvolvimento deste trabalho, bem como, os conceitos fundamentais para se entender os processos envolvidos na metodologia de PBL, dentre esses conceitos temos o entendimento do processo de PBL bem como os procedimentos para realização de um mapeamento sistemático. 


\subsection{Aprendizagem baseada em problemas (PBL)}

A Aprendizagem Baseada em Problemas, ou PBL, é uma metodologia que difere do modelo tradicional de ensino, pelo fato de utilizar problemas de vida real para iniciar o processo de aprendizagem, visando estimular o desenvolvimento de habilidades para solucionar problemas. Esta metodologia preza pelo uso de problemas baseados no mundo real, que são trabalhados em grupos, no qual o problema é usado para iniciar, direcionar, motivar e focar a aprendizagem, ao contrário dos métodos tradicionais que colocam o problema ao final da apresentação do conteúdo (MILTER e STINSON, 1995) e (RIBEIRO, 2005). Ao fazer isso, a PBL apresenta várias mudanças e críticas aos modelos tradicionais de ensino, como aprendizagem colaborativa $\mathrm{e}$ comportamental, pois visa em um aprendizado que resulta em mudança de comportamento e que motiva os alunos melhorando os resultados educacionais (MILTER e STINSON, 1995). Para fornecer uma ampla visão desses estudos, e também pesquisar por estudos que tragam relatos sobre possibilidades de PBL, desafios e outros assuntos afins, é apropriado realizar um Mapeamento Sistemático sobre a temática de PBL.

\subsection{Mapeamento Sistemático}

O estudo de Mapeamento Sistemático (MS) é um método projetado para fornecer uma ampla visão de uma determinada área de pesquisa, que permite identificar, quantificar e analisar os resultados, estabelecendo evidências da mesma sobre um determinado tema (KITCHENHAM, 2007), (ARKSEY e O’MALLEY, 2005).

(PETTICREW e ROBERTS, 2006) afirmam que o MS também pode ser definido como uma pesquisa da literatura, que poderá identificar quais tipos de estudos podem ser abordados por uma Revisão Sistemática, como também indicar o local no qual os mesmos foram publicados, em que bases de dados foram indexados e quais os tipos de resultados apresentaram.

O MS é considerado como um estudo secundário, visto que é uma revisão mais ampla dos estudos primários, tornando-o depende da realização desses estudos para revelar as evidências da pesquisa (KITCHENHAM, 2007). A metodologia utilizada para fazer um estudo de MS é apoiada na mesma visão da metodologia de uma Revisão Sistemática, pois é conduzida de maneira auditável, rigorosa e transparente (ARKSEY e O’MALLEY, 2005). A próxima seção mostra o detalhamento da metodologia utilizada na construção do mapeamento sistemático desse estudo.

\section{Metodologia}

Este trabalho realizou um mapeamento sistemático identificando as principais caraterísticas do uso de PBL na área de Ciência da Computação. Após a avaliação inicial de 2464 artigos, foram selecionados 52 estudos primários relevantes para a pesquisa. A seleção dos 52 estudos primários foi realizada através dos critérios de inclusão e exclusão, conforme apresentado posteriormente.

$\mathrm{Na}$ primeira etapa objetivo foi reunir informações para fornecer uma maior abordagem a cerca da metodologia PBL, possibilitando a compreensão do uso dessa metodologia, e novas estratégias para o ensino da Computação. Durante esse 
procedimento foi realizada a concepção de um protocolo, onde foram descritos o processo e os métodos aplicados na pesquisa. As informações que fazem parte do protocolo serão apresentadas nas subseções seguintes.

\subsection{Questões de pesquisa}

Uma das etapas essenciais do Mapeamento Sistemático foi a definição das questões de pesquisa que conduziram a busca dos documentos relevantes, permitindo posteriormente a triagem de documentos, keywording de abstracts e extração dos dados (PETERSEN, FELDT, et al., 2007). Partindo do objetivo para responder a questão central do estudo que é: "Como se caracteriza a aprendizagem baseada em PBL e qual a sua contribuição e desafios para o ensino da Computação?”.

Foram elaboradas cinco questões secundárias que tiveram como intuito auxiliar nas seguintes respostas:

-QS1: Como a metodologia PBL apoia o ensino na Ciência da Computação?

-QS2: As abordagens existentes de PBL ajudam na formação de profissionais na área de Computação?

-QS3: Quais os critérios utilizados para promover a aprendizagem baseada em PBL?

-QS4: Quais as práticas utilizadas para a aplicação de PBL na Computação?

-QS5: Quais as vantagens e benefícios de se aplicar PBL na Computação?

-QS6: Quais os principais desafios sobre a aprendizagem em PBL na Computação?

\subsection{Estratégia de Busca}

Para a construção dos termos chaves da pesquisa foram utilizadas as palavras-chave da questão central

Quadro1: String de busca

\section{String de Busca}

(learning OR learner OR education OR methodology OR approaches OR "educational program" OR constructivism OR constructivist OR instruction) AND "problem based learning" OR pbl OR "problem based learned") AND (contribution OR advantages) AND (challenges OR outcome OR results) AND (computer OR software OR "computer engineering" OR "information technology" OR "computer science")

\subsection{Fontes de Busca}

A partir da string de busca foi formatada de acordo com as palavras-chaves das questões de pesquisa, e resultou na localização de 2.464 estudos. A quantidade de estudo para cada engenho foi respectivamente: 1242 artigos no IEEExplorer, 181 no Scopus, 327 no Science Direct e 214 no ACM.

\subsection{Seleção dos estudos primários}

Com o objetivo de melhorar os resultados obtidos das buscas nas bibliotecas digitais, foi importante criar alguns critérios de inclusão e exclusão, tendo como base a pergunta de pesquisa definida anteriormente (KITCHENHAM, 2007). 


\section{- Critérios de Inclusão}

Foi considerados todos os estudos que foram encontrados a partir da string de busca nos engenhos citados acima, desde que o estudo esteja disponível na internet e satisfaça alguns dos critérios abaixo: Relevância que o estudo possui em relação à pergunta de pesquisa proposta; Periódicos e artigos completos publicados em revistas, conferências ou simpósios; Estudos que descrevam pesquisas relacionadas ao tema de metodologia de ensino PBL e Ciências da Computação e Estudos que relatem experiências da metodologia favorecendo o ensino no curso de Ciências da Computação.

\section{- Critérios de Exclusão}

Não foi considerados: Estudos não escritos em inglês, visto que para o assunto abordado, existe a possibilidade de não existir artigos publicados em outra língua; Estudos publicados em editoriais, prefácios, artigos de resumo, entrevistas, notícias e revisões; Estudos com resultados experimentais, inconsistentes ou com conteúdo incompleto e Estudos similares (quando dois ou mais artigos apresentam conteúdos semelhantes, foi considerado o estudo mais recente).

\section{Resultados e discussões}

Para a realização da pesquisa, não foi considerado limitação por ano, sendo assim, os Estudos Primários foram publicados entre os anos de 1997 e 2011 . A Figura 1 ilustra a evolução da abordagem de PBL, identificada pelo processo de seleção, ao longo dos anos.

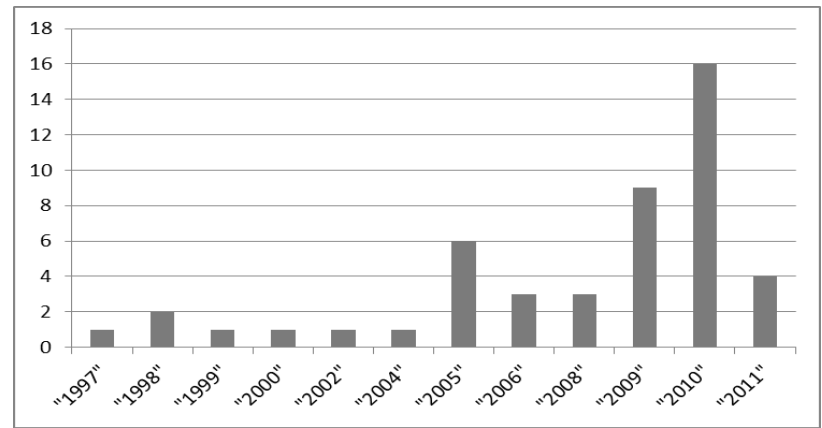

Figura 1: Evolução da abordagem de PBL

Os 52 estudos foram classificados em diversas categorias, de acordo com os assuntos abordado nos estudos. Para a realização da classificação foi feita a leitura dos 52 estudos primários para analisar em qual categoria seria feita a classificação do estudo, e se o assunto abordado seria relevante para o estudo. O quadro 1 mostra as seis categorias e as respectivas quantidades de artigos identificados.

Quadro1: String de busca

\begin{tabular}{lc}
\hline \multicolumn{1}{c}{ CATEGORIA } & QTDE \\
\hline Metodologia PBL & 24 \\
Efetividade & 26 \\
Critérios/Características & 28
\end{tabular}




\begin{tabular}{ll} 
Práticas detalhadas & 33 \\
Benefícios claros & 32 \\
Desafios identificados & 18 \\
\hline
\end{tabular}

Os tópicos abaixo mostram os resultados referentes as seis categorias:

- QS1: Metodologia PBL: Como a metodologia PBL apoia o ensino da Ciência da Computação:

A figura 2, apresenta 8 (oito) tópicos do uso de PBL na Computação, o que mostra que 36\% (9) consideram que para a metodologia ter bons resultados é necessário ter um ambiente colaborativo, fazendo uso de problemas reais, ou que cheguem o mais próximo possível da realidade. Outro tópico relevante foi o destaque para aprendizagem colaborativa, muitos autores acreditam que a metodologia PBL também utiliza conceitos da aprendizagem colaborativa, mas apenas 6 estudos deixaram clara essa evidencia.

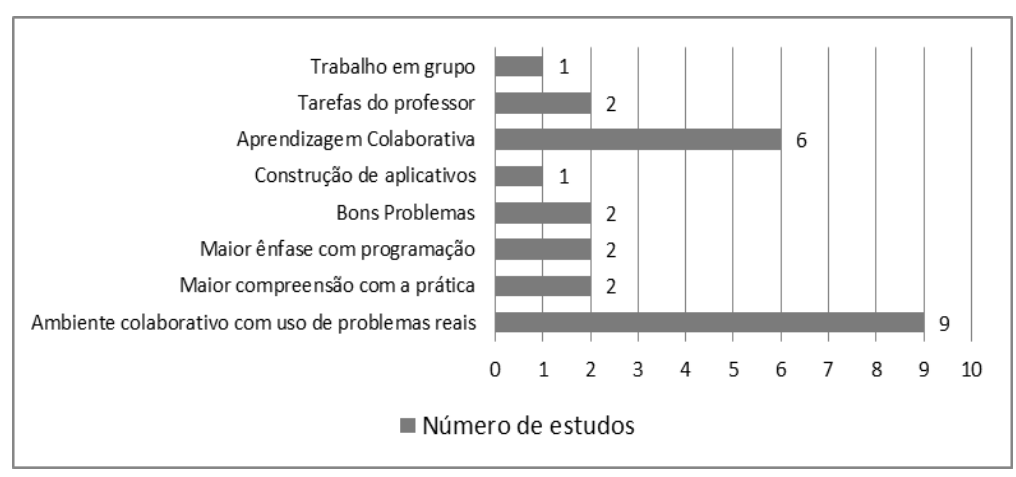

Figura 2: Metodologia PBL

É possível ver que $8 \%$ dos estudos, classificados nessa categoria, mostram bons problemas como sendo fundamental para a aplicação da metodologia.

- QS2: Efetividade: Como as abordagens existentes de PBL ajudam na formação de profissionais da área?

$\mathrm{Na}$ figura 3 pode-se observar 12 tópicos referentes à efetividade da aplicação da metodologia.

Com a maior quantidade de estudos primários, ressaltando que nenhum estudo foi repetido dentre os tópicos, $38,46 \%$ focaram na melhoria em diversas habilidades, ou seja, apresentaram várias habilidades que podem ser desenvolvidas pelos alunos a partir da participação dos mesmos no processo de ensino/aprendizagem. 


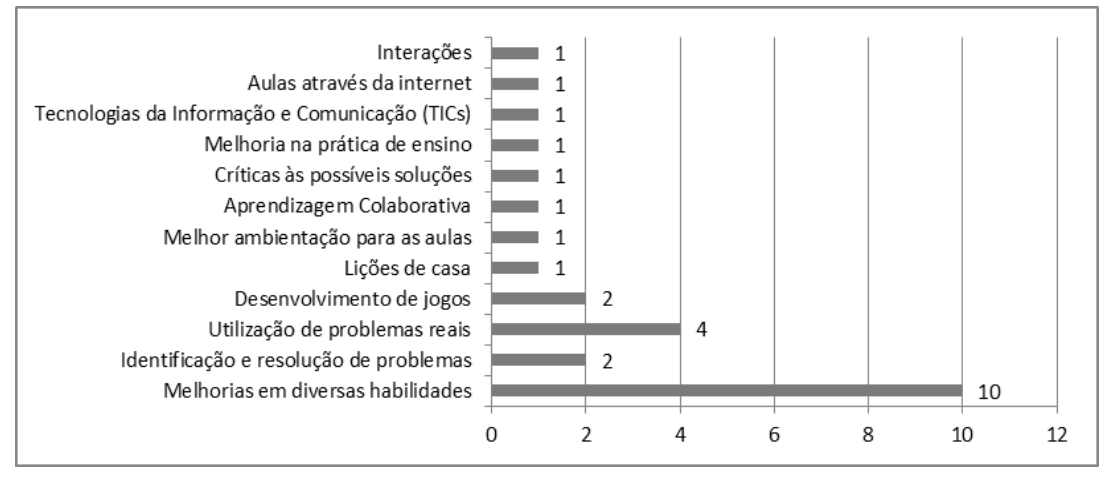

Figura 3: Efetividade

- QS3: Critérios e Características: Quais os critérios utilizados para promover a aprendizagem baseada em PBL.

A figura 4 lista os principais critérios e as principais características da aplicação da metodologia PBL na Ciência da Computação. Dentre esses são destacados 2 (dois) tópicos, os quais tiveram uma maior quantidade de estudos primários. Esses tópicos são: uso de outras abordagens, que mostrou que $45 \%$ dos estudos utilizam como outras abordagens que apoiam a metodologia; e o tópico formação e divisão de grupos, com $44 \%$ dos estudos, ressaltando a importância do trabalho em grupo.

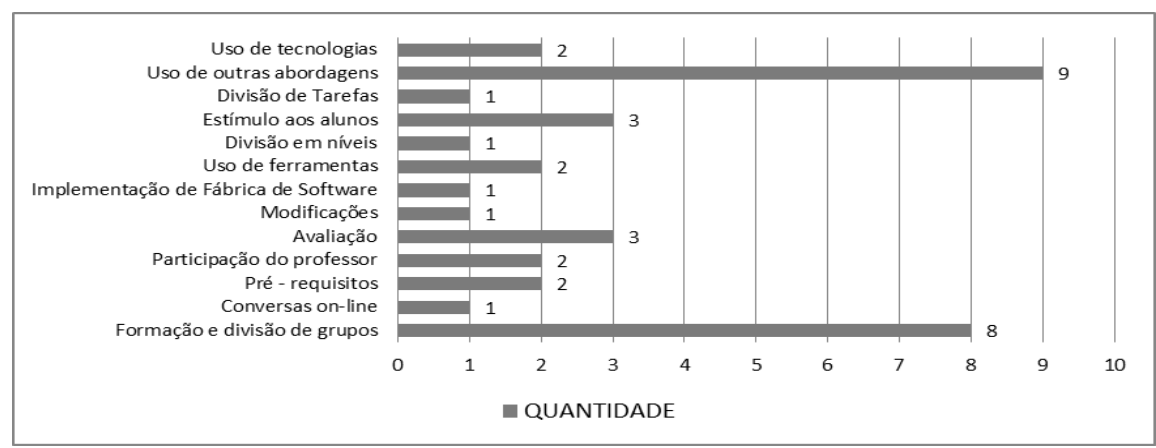

Figura 4: Critérios e Características

- QS4: Práticas Detalhadas: Quais as práticas utilizadas para a aplicação de PBL?

Essa questão buscou investigar como está sendo feita a aplicação de PBL, quais as principais práticas que estão sendo utilizadas no processo de ensino/aprendizagem da área de Ciência da Computação. Como apresentado na Figura 5, 39,47\% dessas práticas são aplicadas nas diversas disciplinas da área, resultando no total de 15 estudos primários que abordaram o assunto. Em segundo lugar, destaca-se o uso de tecnologias ou ferramentas, $21,05 \%$ das práticas realizadas fizeram uso de algum tipo de tecnologia ou ferramenta. 


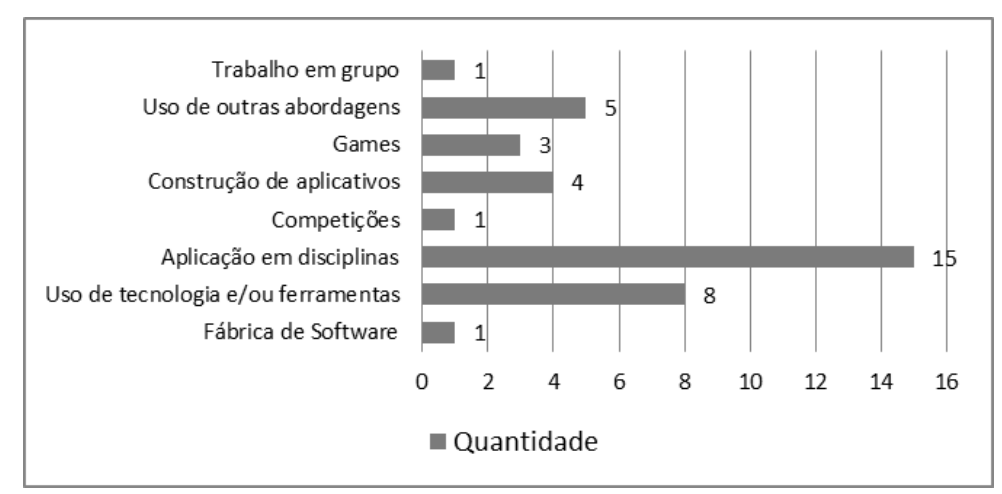

Figura 5: Práticas Detalhadas

- QS5: Benefícios Claros: Quais as vantagens e benefícios de se aplicar PBL na Ciência da Computação?

Essa pergunta tem como objetivo investigar os benefícios da utilização da metodologia na Ciência da Computação. Foram listados diversos benefícios, divididos em 11 tópicos diferentes, que se classificam em ferramentas, desenvolvimento de habilidades, auto avaliação, entre outros. A Figura 6 apresenta todos os tópicos, bem como os estudos primários classificados entre eles. Um dos maiores benefícios resultante do método PBL é o desenvolvimento de diversas habilidades.

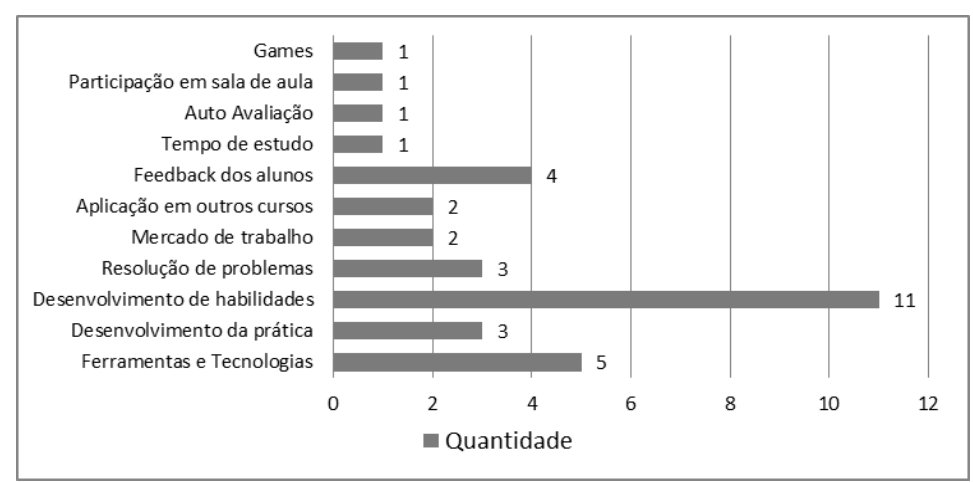

Figura 6: Benefícios Claros

-QS6: Desafios Identificados: Quais os principais desafios sobre a aprendizagem em PBL na Computação?

Essa questão teve o objetivo de listar os principais desafios e problemas encontrados durante o processo de ensino/aprendizagem com PBL na Computação. Foram identificados 12 tópicos que abordam diferentes desafios e problemas. Como pode ser visto na Figura 7, os principais desafios do uso da metodologia é a falta de crença e conhecimento da metodologia, dificuldades nas disciplinas anteriores e aplicação da metodologia. 


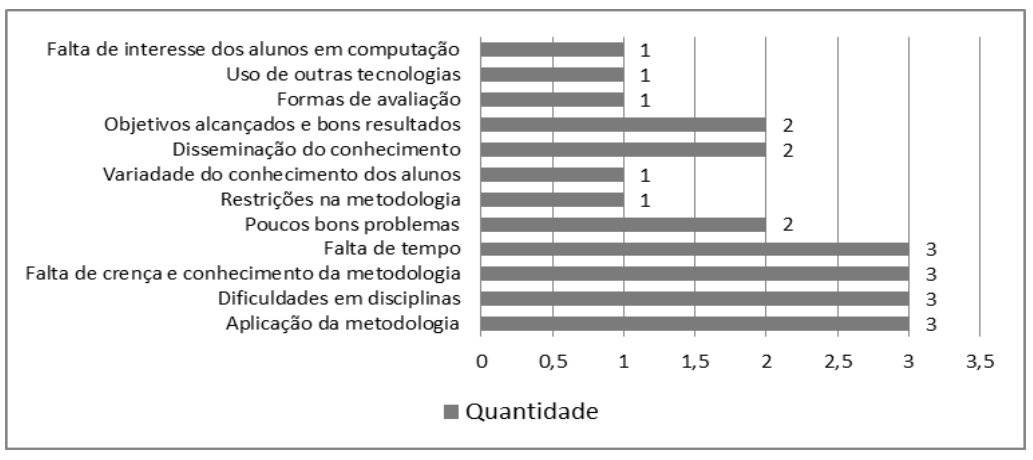

Figura 7: Desafios Identificados

Usando seis questões de pesquisa, o estudo foi capaz de encontrar evidências de forma satisfatória que mostraram que a maioria das aplicações da metodologia de PBL são voltadas para a aplicabilidade nas disciplinas de Engenharia de Software e Programação. O processo de mapeamento foi quantificado e especificado, de forma que torna mais fácil a realização de trabalhos futuros utilizando as evidencias encontradas.

\section{Conclusões}

Dos quatro engenhos de busca utilizados neste trabalho, O IEEE foi o que obteve estudos mais relevantes, totalizando um total de treze estudos primários. Dentre todos os estudos relevantes identificados na pesquisa, os principais benefícios foram, desenvolvimento de diversas habilidades para resolução de problemas reais, trabalho em grupo, autonomia na aprendizagem e aperfeiçoamento das habilidades de comunicação em grupo.

Dentro destas características identificadas, concluímos que a metodologia de PBL vem sendo aplicada na área de ciência da computação juntamente com o uso de outras metodologias, ou seja, são utilizados diferentes métodos em conjunto com PBL, facilitando a melhoria do ensino e a formação de profissionais com uma visão mais ampla dos problemas reais na área de Ciência da Computação. Dentre os desafios identificados no decorrer desta pesquisa, podemos dizer que o mais impactante tenha sido relacionado com as buscas realizadas. O estudo se baseou em quatro engenhos e o processo de filtragem foi automatizado, é possível que estudos relevantes possam ter ficado de fora dessa filtragem inicial, tendo em vista que cada engenho tem suas próprias regras de extração e classificação de artigos, independente da utilização da mesma string de busca em todos os engenhos.

Tendo em vista que existem diferentes abordagens pedagógicas no processo de ensino e aprendizagem, como trabalho futuro, pretendemos identificar as abordagens já consolidadas e que possam ser utilizadas junto à metodologia de PBL. Para tal será feito um apanhado de diferentes abordagens já existentes e identificado características em comum com a metodologia de PBL. 


\section{Referências}

Andrade, G. P. et al. (2007). "Aplicação do Método PBL no Ensino de Engenharia de Software: Visão do Estudante”.

Arksey H. and O’Malley L. (2005). “Scoping Studies: Towards A Methodological Framework".

Coles, C. R. (1991). 'Is problem-based learning the only way?”.

Deslisle, R.(1997)."How to use problem-based learning in the classroom.” Alexandria: ASCD.

Kitchenham, B. (2007).’Guidelines for performing Systematic Literature Reviews in Software Engineering".

Martin, K. and Chinn, D.(2005). "Collaborative, Problem-Based Learning in Computer Science".

Milter, R. G. and Stinson, J. E. (1995). 'Educating leaders for the newcompetitive environment".

Petersen, K. et al. (2007). "Systematic Mapping Studies in Software Engineering”.

Petticrew, M. and Roberts, H.(2006.) "Systematic Reviews in the Social Sciences: A Practical Guide".

Ribeiro, L. R. C. (2005). "A aprendizagem baseada em problemas (PBL): Uma implementação na educação em engenharia na voz dos atores".

Santos, S. C. et al. (2010). “Applying PBL in Software Engineering Education”.

Savin-Baden, M. (2000). "Problem Based Learning in Higher Education: Untold Stories".

Schmidt, H. G. (1993). 'Foundations of Problem-Based Learning: Some Explanatory Notes".

Yates, W. R. and Gerdes, T. T. (1996). 'Problem Based Learning in Consultation Psychiatry". 